\title{
Outcomes analysis-the role of case management
}

\author{
This article was published in the following Dove Press journal: \\ Journal of Healthcare Leadership \\ 30 April 2010 \\ Number of times this article has been viewed
}

\author{
Marietta Stanton \\ Donna Packa \\ Capstone College of Nursing, \\ Tuscaloosa, AL, USA
}

Correspondence: Marietta Stanton Capstone College of Nursing, Box 870358, Tuscaloosa, AL, USA $\mathrm{Tel}+\mathrm{I} 205348$ I020

Fax + I 2053485559

Email mstanton@bama.ua.edu

\begin{abstract}
The purpose of this presentation is to examine the potential role of case management in evidence, in outcomes measurement and analysis. Case management at the individual patient care or microsystem level and at the organizational or macrosystem level are both necessary for an effective outcomes management program. The preparation and experience of case managers at both levels of practice are examined. Outcomes and outcomes management procedures as delineated in the literature are used as a framework for differentiating the two levels of practice and preparation of case managers.
\end{abstract}

Keywords: case management, outcomes analysis, macrosystem, microsystem

\section{Introduction}

Case management has been defined "as a collaborative process of assessment, planning, facilitation and advocacy for options and services to meet an individual's health needs through communication and available resources to promote quality cost-effective outcomes."1 Outcomes measurement includes: principles of continuous quality improvement/performance improvement (CQI/PI); use of benchmarks; analysis of trends; incorporation of evidence based practice; processes which promote the understanding of current condition; outcomes; and the examination of return on investment related to a specific intervention. ${ }^{2}$ Case managers can cooperate with the health care team to incorporate best practices, assess outcomes at the level of individual patient care or at the point of service; and they can also design, monitor, evaluate and manage aggregate outcomes at the macrosystems level, as a case management administrator. Although case managers work in a variety of health care settings, for example, primary, secondary, tertiary and restorative care, the use of best evidence and the assessment of health care outcomes is a central component of the role. Essentially the broad categories of outcomes for monitoring and management are very similar although vary in breadth, complexity and scope of data and analysis. This presentation focuses on the concept of outcomes management at two levels of case management practice; at the individual, point of service or microsystems level, and the macrosystems or organizational level.

Case management at the basic or patient care level should be coordinated by individuals who are experienced clinicians. In nursing and other health professions, preparation at the entry level of practice, continuing education in case management principles and experience provide the appropriate skill set required at this microsystem level. To validate or document this skill set, an individual can be certified in case management by national, accredited certifying bodies. For example, the certified case manager (CCM) is a well respected certification that is recognized across disciplines 
and settings. ${ }^{3}$ Case management administrators or those who coordinate case management at the organizational or macrosystems level should have all the qualifications of those practicing at the individual patient care level and in addition they should also have graduate academic preparation in case management or clinical resource management and leadership. These individuals can also be certified as administrators in their field and have certification in case management administration. ${ }^{4}$

There is no doubt that case management has an impact on outcomes management. A review of the recent literature in the field of case management demonstrates that case management has an impact on outcomes across diverse health care settings. Engelke and colleagues demonstrated that case management was effective in not only tracking but enhancing outcomes in the school health setting. ${ }^{5}$ Clinical outcomes were effectively monitored and managed using case management at patient care level. ${ }^{6}$ Bender illustrated the effectiveness of case management in monitoring outcomes for a Medicare population. ${ }^{7}$ Similarly, the use of case management was an effective strategy for improving program performance for people living with HIV/AIDS. ${ }^{8}$ Cotter and colleagues found case management was effective in enhancing activities associated with transitions of care. ${ }^{9}$ Sutherland and Hayter evaluated and substantiated the effectiveness of case management in improving outcomes for three major chronic diseases, diabetes, chronic obstructive lung disease and coronary artery disease. ${ }^{10}$ There is extensive additional information in the literature to document the efficiency and effectiveness of case management in outcomes management, improved outcomes, and the use of best evidence to determine outcomes. To effectively monitor outcomes in any given agency, two levels of analysis are necessary for effective outcomes management. These two levels include monitoring health outcomes at the point of care and at the organizational level. ${ }^{8}$

Health care outcomes at the point of care can be measured using clinical, functional, financial, quality and patient satisfaction indicators. Outcomes at the organizational level include: aggregate clinical; functional; and quality outcomes; as well as costs of care; lengths of stay; re-hospitalizations; use of acute services; cost benefit; return on investment; and satisfaction rates for different patient populations. ${ }^{8}$ Aggregate data regarding outcomes are most effectively monitored and managed at the organizational level by a case management administrator. ${ }^{4,11}$ It is contended that skills set for the two different case management roles are essential although they differ in: complexity; require distinct skill sets; and suggest different levels of academic preparation and focus, in order to maximize the outcomes of the management process.

\section{Case management at the microsystems level}

At the microsystems level, or the point of care, case managers evaluate clinical, satisfaction, functional, selected financial and other quality outcomes of individual patients. ${ }^{12,13}$ They share this responsibility with the interdisciplinary team but case managers have the proper skill set through their basic academic preparation, clinical experiences and continuing education to serve as the nexus for the coordination of outcomes from admission through to discharge and beyond, through various transitions of care. ${ }^{13}$ During these health care transitions from one setting to another, case managers are positioned to assure that serious lapses in care do not transpire. ${ }^{14}$ Although the traditional models of case management focus on outcomes for hospital care, case managers at the point of service can be positioned to promote the desired outcomes in diverse clinical settings such as: health promotion; disease prevention; public health and primary care programs; as well as chronic; restorative; and rehabilitation care. To participate in outcomes measurement at the point of service or patient care level, case managers are often guided by protocols, guidelines, tool kits or algorithms pertinent to the patient's clinical situation. Patient, provider and system variances from these protocols are determined and best practice interventions are used as a trajectory to deal with a clinical condition and identify "outliers". Likewise functional outcomes may be guided by tools that focus on activities of daily living and other dimensions pertinent to patient performance and well being. Case managers evaluate patient satisfaction with their care and providers using qualitative data, such as feedback from the patient, family and significant others, as well as quantitative data monitored through a preselected tool, ie, Picker/Commonwealth, Patient Satisfaction Survey. Patient satisfaction in today's health is an important aspect of case management. The Agency for Health Care Policy and Research (AHCPR), conducted a five-year study; The Consumer Assessment of Health Plans Study (CAPHS), to provide an integrated set of carefully tested and standardized survey questionnaires and report formats that would provide accurate, meaningful and reliable information about patient experiences and satisfaction with their health plans. This survey, and others that measure patient satisfaction, help case managers understand patient choices, preferences and behaviors. ${ }^{15}$ Using these surveys along with qualitative data collected during patient interaction with the case manager, 
provide an avenue for discussing patient concerns with the interdisciplinary team so that modifications in the treatment plan can be accommodated.

Financial outcomes are also monitored at the point of service including: length of stay; preexisting or hospital acquired conditions; mechanisms for reimbursement of care costs; and the appropriate utilization of services in the hospital or care in other settings. Careful monitoring of preexisting conditions is critical to ensure that a particular clinical condition is not hospital acquired.

In setting such disease management programs, the case manager positioned to intervene with individual clients contributes significantly to monitoring patients with the most costly care, to keep these costs within an acceptable range and avoid unnecessary hospitalizations. Case management within the microsystem is often focused on high-risk conditions across a wide variety of settings.

In a health promotion environment, case managers monitor health behavior goals and, in disease prevention, they help patients adjust their life style to reduce risk behaviors and to focus on problem areas in their health profile. Satisfaction, with care, functional and clinical status and financial outcomes can be delineated for health promotion and disease prevention using Healthy People 2010 to provide guidance for potential outcome formulation in these areas.

In restorative or chronic care, the Centers for Medicare and Medicaid Services reported that $54 \%$ of all health care dollars are spent within the last two years of life. However, the data also indicate that the higher cost in health care dollars spent near the end of life do not equate to improved quality of care. ${ }^{16}$ Therefore, in the chronic care arena case managers at the point of service can have a major impact on outcomes of care. Case managers have the capacity to balance quality and cost in many health care settings, and particularly in the case management of patients with chronic health problems near the end of life.

Outcomes of care may also be assessed using benchmarks to determine where the agency's outcomes rank in relation to other similar agencies, services or national standards. These benchmarks may be generated within the agency or by an external agency such as the Joint Commission. Benchmarks may also assist case managers to focus on care that meets or exceeds these standards. Use of such standards for comparison, are critical to outcomes measurement in any setting.

Case managers at the point of service participate in performance improvement and CQI so that adjustments in care can be made expediently when problems occur, and over a longer period of time, by modifying plans of care for a particular health problem. Appropriate systems must also be in place for data collection at the point of service. Case managers may also be involved in assessing whether clinical documentation at the point of service provides the adequate information about patient's situation. This in turn has an impact on both reimbursement and rates of reimbursement, and therefore on the financial outcomes of the system. Documentation of outcomes needs to be carefully audited to ensure that providers include appropriate information in the patient record.

It is clear that case management at the basic level of practice are the eyes and ears of quality care. Not only do case managers assess, implement, monitor and evaluate outcomes, they are in a position to alter the plan of care in collaboration with providers and to adjust care so that benchmarks and standards are met. The role of the case manager within the micro-system is not to determine the overall outcomes of care, but to monitor and evaluate how care at the point of service meets those predetermined outcomes.

In summary, case managers at the point of service or individual patient care level assess, monitor, and participate in the evaluation of outcomes. They are in the proverbial trenches coordinating care, collecting, documenting, monitoring data, and consistently using benchmarks to determine if predetermined outcomes are met. Case managers assess outcomes and document the data needed to evaluate the achievement of them. They participate in gathering best evidence and seek coordination of the health care team to facilitate achievement of outcomes. Case managers are familiar with the measurement tools and instruments used to monitor financial, functional, and clinical status satisfaction with care and quality outcomes. They monitor clinical documentation and ensure that procedures used at the point of service meet the expectations of accrediting and regulatory agencies. They manage and monitor transitions of care. Case managers at this level must achieve peak performance by building upon their basic education through continuing education and clinical experience in their practice area. They adhere to the standards of practice for case management regardless of the setting and they seek certification at the appropriate level to demonstrate to providers and patients that they are qualified to practice case management. They are familiar with standards of care and guidelines for their clinical area. ${ }^{1}$ They participate in $\mathrm{PI} / \mathrm{CQI}$ initiatives and serve on quality management committees. Thus, case managers are essential to outcomes measurement at the microsystems level.

\section{Case management at the macrosystems level}

Case management is also important at the macrosystems or organizational level and involves the coordination and 
management of the entire case management structure and process used within an agency. ${ }^{8}$ At this level, clinical, financial, quality, satisfaction and functional outcomes are also monitored and reported, yet focus on aggregate data for entire populations or disease management programs. Data collected at the point of service are analyzed to provide information about selected outcomes for entire cohorts, populations and to determine trends, participate in clinical resource management decisions and monitor return on investment (ROI). The case manager at the organizational level of outcomes management needs a more complex skill set than is needed at the microsystems level.

The Credentialing Advisory Board for Case Management Administrators Certification has named seven competency domains ${ }^{2}$ including:

- Identification of at risk populations

- Assessment of clinical systems and looking at gaps in service requirements

- Development of strategies to manage populations

- Providing leadership for change

- Market assessment and strategic planning

- Human resource management

- Program evaluation

The case management administrator should have a graduate degree in the health professional discipline, ie, nursing, and the content and focus of that advanced preparation should include: leadership; management; finance; human relations management; strategic planning; and marketing. Case management administrators also require knowledge regarding: population health; examining population aggregates or disease management populations; determining trends; and implementing predictive modeling concepts and approaches. Experience at or an in-depth knowledge of case management at the microsystems level of case management is also an imperative foundation for any case management administrator. To fully coordinate outcomes management at the macrosystems level, the case manager administrator must be able to design, implement and evaluate protocols of care using best evidence, in collaboration with other health care leaders; and that implies a skill set well beyond what can be achieved through basic entry level academic preparation, experience, and continuing education.

At the administrative level or organizational level, outcomes are defined and derived from the data produced at the microsystems level. Not only are data aggregates used to formulate, revise and evaluate outcomes, but best practices and evidence from a variety of sources are used based on the clinical condition or setting. This may involve the examination of peer reviewed journals or databases like CINAHL or PubMed. The National Guidelines Clearinghouse is a free resource for protocols and treatments for a variety of health conditions including medical and behavioral health issues. ${ }^{17}$ Case managers at this advanced level should be able to examine evidence from randomized control trials, systematic reviews and other high level evidence to develop, in conjunction with health team members, appropriate protocols and related outcome measures.

Tools and instruments to measure outcomes may be generic health status measures, condition specific or a combination of the two. These tools are often used in health care outcomes research, which in turn, generates outcome measures for the clinical arena. These tools and instruments have also been used in practice to gather information and measure outcomes. ${ }^{15}$ Case management administrators must be prepared to conduct applied research as well as performance improvement projects. Clinical protocols, toolkits, and guidelines are the foundation of any multidisciplinary evaluation of outcomes. The case management administrator identifies these protocols or assists in their development and evaluation, based upon research findings in the literature. These are then implemented at the individual patient care level for monitoring by the case manager functioning at the basic level of practice. Case management administrators at this level perform root cause analysis to protect patient safety and deal with medical issues and provide the performance indicators or "dashboard" for monitoring at the point of service or care. ${ }^{18}$

Strategies for meaningful outcome assessment at this macrosystem level include identifying outcome indicators that provide useful information for large numbers of patients. The selected outcome indicators must be feasible and relatively inexpensive to obtain. These outcome indicators must be comparable across populations and providers, and they must be uniformly collected. The indicators may need adjustment according to patient characteristics. The idea is to collect outcome indicators on large samples of patients and compare both expected and actual outcomes. ${ }^{19}$ Outcome measurement, performance monitoring and quality improvement are data-driven endeavors that are critical to case management administrators and are made more efficient by selecting the proper software tools for data analysis. This is an integral component of case management at this advanced level. Case managers at the administrative level need to be familiar with software used to capture outcomes data. The case management administrator monitors system levels, provides case management as well as patient aggregate 
data. In many hospitals, the case management administrator is responsible for the coordination of clinical documentation monitoring and, therefore, the monitoring of reimbursement. The structure, process and outcomes of the case management system are modified as a result of outcomes analysis at this level.

The case management administrator is like the coach or quarterback in a football game. The administrator, like the quarterback, knows the plays and the overall game plan and directs the other players specifically, ie, the point of care case managers, to provide a system of outcomes management. The physician and health care providers like assistant coaches participate in the development of the game plan and yet it is the quarterback that executes the plan with the other players. Effective outcome assessments are dependent on case managers doing their job at both levels of case management practice. However, if the case management function is not supported and respected both outcome assessment and management are difficult, if not impossible, in any organization. It is critical that the case management administrator not only monitors the outcomes associated with the quality of care but also monitors the qualities and outcomes of the case management system itself. The case management administrator should not only seek credentialing for his/her own practice they should also seek accreditation for the entire case management service.
The case manager should therefore have a key position on the executive management team.

Figure 1 depicts a model for outcomes management using the two levels of case management described in this presentation. The model reflects these two levels of practice and the specific outcomes and activities appropriate at each level of case management.

\section{Summary}

In summary, the two levels of case management have been presented for the effective management of outcomes. The approach to outcomes management using both the individual and administrative level of case management practice can be used as a template in a wide variety of settings and across primary, secondary and tertiary levels of practice. The necessity for different levels of education and experience for both point of care and administrative case managers is justified based on the different skill sets required. The approach to outcomes management is different for both, in that case managers at the point of service or microsystem level are focused on care coordination and the collection of data concerning financial, clinical, satisfaction, functional and quality outcomes of individual clients. These data are provided to the case management administrator who analyzes the data, adjusts protocols and approaches, and provides

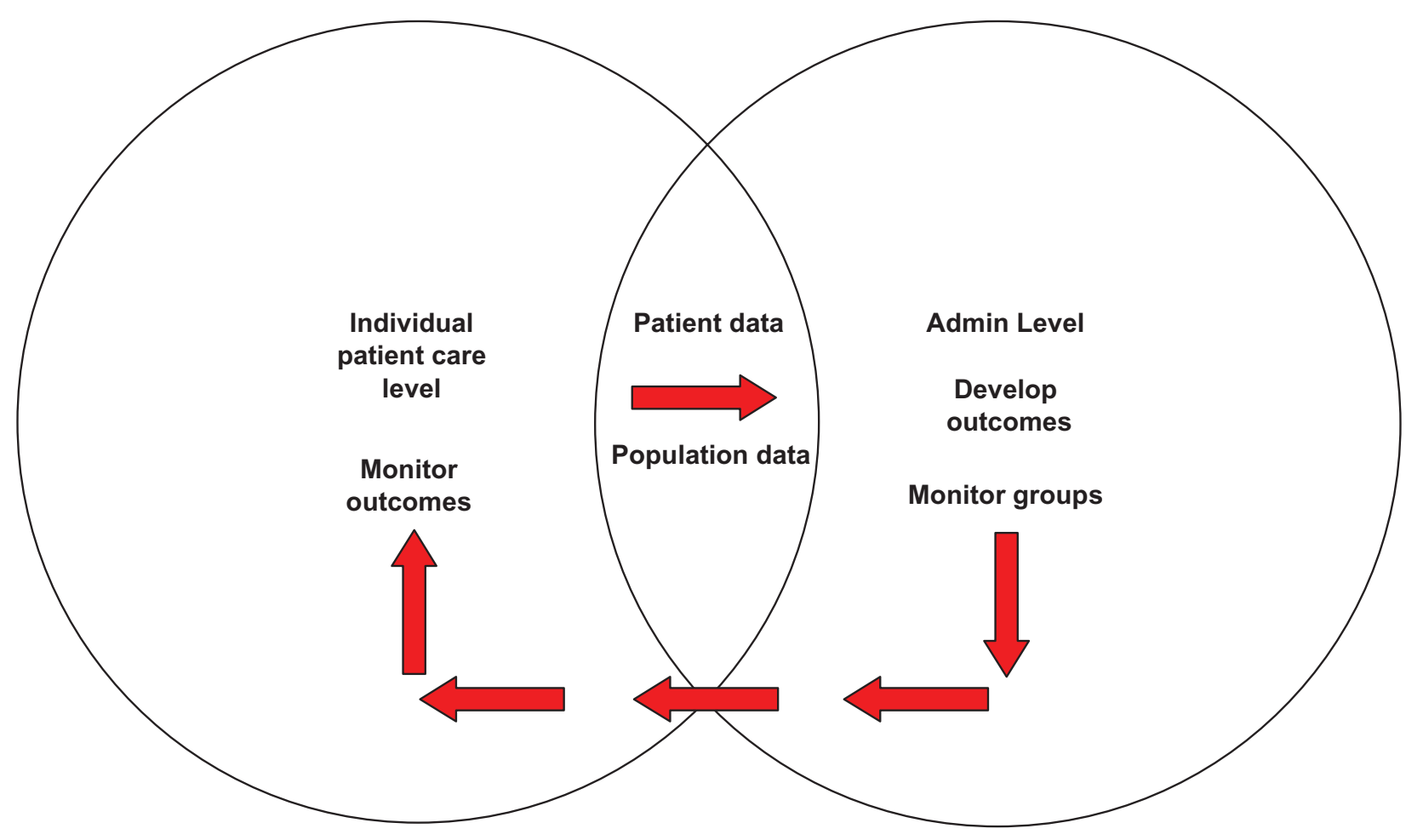

Figure I Outcomes management and case management practice. 
feedback about the outcomes and related indicators to the point of care case managers.

Case management is integral to outcomes management and there are many dimensions to outcomes analysis. Technology and software are available to provide feedback about care provision, cost and quality. However, the real impetus for serious outcome assessment is best accomplished with a dedicated and committed case management system at both the micro- and macrosystems levels. Case management is not a functional part of another role; it is a distinct and separate role, with specific skill sets at both the micro- and macrosystem levels. Case management is not a part-time responsibility; rather, it is a full time focus on outcomes, quality and cost. The most effective outcomes assessment is achieved in systems that have a high quality, dedicated system of case management.

\section{Disclosure}

The authors reports no conflicts of interest in the work.

\section{References}

1. Case Management Society of America. Standards of Practice for Case Management. Little Rock, AR: Case Management Society of America; 2002.

2. Zander K. Hospital Case Management Models: Evidence for Connecting the Boardroom to the Bedside. Marblehead: HCPro, Inc.; 2008.

3. Commission for Case Manager Certification 2010. Available from: http:// www.ccmcertification.org/pages/123body.html. Accessed on January 10, 2010.

4. Stanton MP, Swanson M, Sherrod R, Packa D. Case management evolution: From basic to advanced practice role. Lippincott's Case Management, The Journal of Professional Practice. 2005;10(6):274-287.

5. Engelke MK, Guttu M, Warren MB. Defining, delivering, and documenting the outcomes of case management by school nurses. The Journal of School Nursing. 2009:25(6);417-426.
6. Malakouti SK, Nojomi M, Panaghi L, et al. Case management for patients with schizophrenia in Iran: A comparative study of the clinical outcomes of mental health workers and consumers' family members as case managers. Community Mental Health Journal. 2009:45(6); 447-452.

7. Bender, M, Nancee L. An analysis of the processes and outcomes of coordination of care: A home care organization initiated case management intervention in a medicare population. University of Rochester School of Nursing. 2003:AAT(3120691);1-296.

8. Lehrman S, Gimbel R, Freedman J, Savicki K, Tackley L. Development and implementation of an HIV/AIDS case management outcomes assessment programme. AIDS Care. 2002:14(6);751-761.

9. Cotter JJ, Smith WR, Boling PA. Transitions of care: The next major quality improvement challenge. British Journal of Clinical Governance. 2002:7(3);198-206.

10. Sutherland D, Hayter M. Structured review: evaluating the effectiveness of nurse case managers in improving health outcomes in three major chronic diseases. Journal of Clinical Nursing. 2009;18(21):2978-2992.

11. Noetscher CM. Using data in the case management process. Journal of Nursing Care Quality. 1999:1;86-97.

12. Stanton M, Packa D. A model for rural case management. Journal of Case Management. 2001;2(6):96-103.

13. Stanton M, Dunkin J. A retrospective analysis of case management, functions, roles and responsibilities at a nurse managed clinic. Professional Case Management. 2009;14(6):321-327.

14. Parrish MM, O’Malley K, Adams RI, Adams SR, Coleman EA. Implementation of the care transitions intervention: Sustainability and lessons learned. Professional Case Management. 2009;14(6):282-293.

15. Kane RL. Understanding Health Care Outcomes Research. Sudbury, MA: Jones and Bartlett Publishers; 2004.

16. Owen M. The heartbeat of case management: Now is the time. Professional Case Management. 2010;15(1):45-46.

17. Stanton MP. Shortening the case management learning curve: A call to action. Professional Case Management. 2009;14(6):278-279.

18. Smith S. Cultivating a Culture of Safety in healthcare: A Systematic Approach to Root Cause Analysis. Cullman: QIG Publishing; 2008

19. Parsons ML, Murdaugh CL, Purdon TF, Jarrell BE. Guide to Clinical Resource Management. Gaithersburg: An Aspen Publication; 1997.
Journal of Healthcare Leadership

\section{Publish your work in this journal}

The Journal of Healthcare Leadership is an international, peer-reviewed, open access journal focusing on leadership for the health profession. The journal is committed to the rapid publication of research focusing on but not limited to: Healthcare policy and law; Theoretical and practical aspects healthcare delivery; Interactions between healthcare and society and evidence-based practices;

\section{Dovepress}

Interdisciplinary decision-making; Philosophical and ethical issues; Hazard management; Research and opinion for health leadership; Leadership assessment. The manuscript management system is completely online and includes a very quick and fair peer-review system. Visit http://www.dovepress.com/ testimonials.php to read real quotes from published authors. 